NASA/TM—2009-215183

\title{
Morphology-Dependent Resonances and Their Applications to Sensing in Aerospace Environments
}

\author{
G. Adamovsky
}

Glenn Research Center, Cleveland, Ohio

M.V. Otugen

Southern Methodist University, Dallas, Texas 


\section{NASA STI Program . . . in Profile}

Since its founding, NASA has been dedicated to the advancement of aeronautics and space science. The NASA Scientific and Technical Information (STI) program plays a key part in helping NASA maintain this important role.

The NASA STI Program operates under the auspices of the Agency Chief Information Officer. It collects, organizes, provides for archiving, and disseminates NASA's STI. The NASA STI program provides access to the NASA Aeronautics and Space Database and its public interface, the NASA Technical Reports Server, thus providing one of the largest collections of aeronautical and space science STI in the world. Results are published in both non-NASA channels and by NASA in the NASA STI Report Series, which includes the following report types:

- TECHNICAL PUBLICATION. Reports of completed research or a major significant phase of research that present the results of NASA programs and include extensive data or theoretical analysis. Includes compilations of significant scientific and technical data and information deemed to be of continuing reference value. NASA counterpart of peer-reviewed formal professional papers but has less stringent limitations on manuscript length and extent of graphic presentations.

- TECHNICAL MEMORANDUM. Scientific and technical findings that are preliminary or of specialized interest, e.g., quick release reports, working papers, and bibliographies that contain minimal annotation. Does not contain extensive analysis.

- CONTRACTOR REPORT. Scientific and technical findings by NASA-sponsored contractors and grantees.

- CONFERENCE PUBLICATION. Collected papers from scientific and technical conferences, symposia, seminars, or other meetings sponsored or cosponsored by NASA.

- SPECIAL PUBLICATION. Scientific, technical, or historical information from NASA programs, projects, and missions, often concerned with subjects having substantial public interest.

- TECHNICAL TRANSLATION. Englishlanguage translations of foreign scientific and technical material pertinent to NASA's mission.

Specialized services also include creating custom thesauri, building customized databases, organizing and publishing research results.

For more information about the NASA STI program, see the following:

- Access the NASA STI program home page at http://www.sti.nasa.gov

- E-mail your question via the Internet to help@ sti.nasa.gov

- Fax your question to the NASA STI Help Desk at 301-621-0134

- Telephone the NASA STI Help Desk at 301-621-0390

- Write to: NASA Center for AeroSpace Information (CASI) 7115 Standard Drive Hanover, MD 21076-1320 
NASA/TM-2009-215183

\section{Morphology-Dependent Resonances and Their Applications to Sensing in Aerospace Environments}

G. Adamovsky

Glenn Research Center, Cleveland, Ohio

M.V. Otugen

Southern Methodist University, Dallas, Texas

National Aeronautics and

Space Administration

Glenn Research Center

Cleveland, Ohio 44135 


\section{Acknowledgments}

The work has been supported by the Integrated Vehicle Health Management, IVHM, Project under NASA Aviation Safety

Program. M.V. Otugen gratefully acknowledges the support from NASA Glenn Research Center, (NASA Grant NAG3-2679), and the National Science Foundation (through grants CTS-0502421, IIP-0539067, and CBET-0619193).

Level of Review: This material has been technically reviewed by technical management.

Available from

NASA Center for Aerospace Information 7115 Standard Drive

Hanover, MD 21076-1320
National Technical Information Service 5285 Port Royal Road Springfield, VA 22161

Available electronically at http://gltrs.grc.nasa.gov 


\title{
Morphology-Dependent Resonances and Their Applications to Sensing in Aerospace Environments
}

\author{
G. Adamovsky \\ National Aeronautics and Space Administration \\ Glenn Research Center \\ Cleveland, Ohio 44135 \\ M.V. Otugen \\ Southern Methodist University \\ Dallas, Texas 75275
}

\begin{abstract}
This paper reviews recent developments in Morphology — Dependent Resonance (MDR) — based sensors for aerospace applications. The sensor concept is based on the detection of small shifts of optical resonances (also called the whispering gallery modes or WGM) of dielectric spheres caused by external effects. Recent developments in MDR-based micro-optical sensors for temperature, force, pressure and concentration are discussed. In addition to the experimental configurations used in each type of prototype sensor, a brief overview is also given for analytical approaches to describe the sensor principle.
\end{abstract}

\section{Nomenclature}

F force in Newtons

$l \quad$ polar mode number

$m \quad$ azimuth mode number

$n \quad$ radial mode number

$n_{S} \quad$ refractive index of sphere

$P_{0} \quad$ pressure in Pascals

$r \quad$ radial dimension or radial argument in spherical coordinates

$R_{S} \quad$ radius of a microsphere

$t \quad$ time

$\alpha \quad$ thermal expansion coefficient

$\beta \quad$ thermo-optic coefficient

$\lambda \quad$ vacuum wavelength of light

$\Delta n_{s} \quad$ change in refractive index of a microsphere

$\Delta R_{s} \quad$ change in the radius of a microsphere

$\Delta T \quad$ change in temperature

$\Delta \lambda \quad$ change in the wavelength of light

$\Delta \lambda_{\text {FSR }} \quad$ free spectral range

$\Delta \lambda_{l} \quad$ spacing between two polar modes $l$ in wavelength domain

$\theta \quad$ polar angle in spherical coordinates

$\varphi \quad$ azimuth angle in spherical coordinates

\section{Introduction}

Dielectric microspheres (or similar geometries) are optical structures that exhibit resonant properties, meaning they can select very narrow segments of the incoming signal's spectrum for further manipulation and processing. The optical resonances of a microsphere are frequently called the whispering gallery 
modes (WGMs). In general, microspheres belong to the same group of devices as Fabry-Perot interferometers and fiber Bragg gratings. The optical resonances in microspheres are a function of their morphology, meaning, their geometry, and dielectric properties (refractive index). Any perturbation to their morphology (shape, size or refractive index) caused by a change in the surrounding environment will lead to a shift in the resonances (WGM). By tracking these morphology-dependent shifts of WGMs, it is possible to measure the change in a given environmental property. In comparison to Fabry-Perot interferometers and fiber Bragg gratings, MDR microsphere sensors can be built in smaller sizes and they tend to exhibit significantly higher quality factors, $Q$. The small size permits the use of these microspheres in a very dense fashion on a very small footprint, so compact multipurpose devices for sensing could be constructed. On the other hand, the high quality factor defined as $Q=\lambda / \Delta \lambda$, where $\lambda$ is the wavelength at which a resonance occurs and $\Delta \lambda$ the linewidth of the resonant wavelength, offers the potential of very high sensitivity of the measured quantity. $Q$-values as high as $10^{9}$ have been reported in the literature (ref. 1).

Devices that employ high $Q$-value MDRs have been reported in the literature with applications primarily to communications and, more recently, some research has focused on sensing applications including biological sensors. In communication, MDR-based channel dropping filters (ref. 2) and modulators (ref. 3), among others (ref. 4), have been demonstrated. Further, using the recent advances in microlithography, several MDR-based devices have been made on a common substrate (ref. 5) permitting coupling signal at resonant modes from one resonator to another. In these applications, the resonator is perturbed externally to affect a change in its morphology. In the sensor application, the MDR device is a passive element; the change in a specific environmental condition perturbs the morphology of the resonator which is measured quantitatively by monitoring the resonance shifts. In the case of biological sensing, typically, the optical resonance shifts occur due to changes in the physical conditions of the surrounding medium alone (without inducing a perturbation to the morphology of the resonator itself). For example, a change in the refractive index of the surrounding medium alone will have an effect on the structure of sphere resonances and may be used to detect the presence of a certain chemical or a biological agent. Recently, several applications of this type have been explored including protein detection (refs. 6 to 8 ).

This paper will focus on the applications of MDRs to sensing parameters relevant to the control and health management of aerospace vehicles.

\section{Principle of Operation}

The morphology dependent resonances (MDR), also known as whispering gallery modes (WGM), of spheres have been the subject of a number of theoretical studies. Techniques used to describe the phenomenon could be loosely grouped into three broad categories. The first group of analytical techniques uses Maxwell's equations and propagates the incident fields through a medium that includes resonating objects or cavities (refs. 9 to 11). Within a homogeneous sphere the electromagnetic field is expressed in terms of its components in spherical coordinates (refs. 12 to 14). The process of solving the

Maxwell equations in spherical coordinates and solutions that result from the process have been described in the literature (refs. 15 and 16). The analytical techniques derived from solving Maxwell's equations could be somewhat cumbersome; however they permit the introduction of polarized electromagnetic fields (refs. 12 and 17).

The other group contains methods and techniques of quantum mechanics, such as the potential well principle among others (refs. 18 to 20). These methods use quantum-mechanical analogy between the scalar Helmholtz equation that results from solving the Maxwell equations in spherical coordinates and the Schrödinger equation (ref. 20). A Schrödinger-like equation and analogy with the hydrogen atom have brought a concept of the photonic atom model (refs. 21 and 22). The photonic atom model also helps to analyze the quantization process. Techniques based on the potential well theory are useful since they may provide a clearer understanding of the effects of constituent parameters of the resonators and the incident field. 
Finally, the last group includes a geometric optics approach with some modifications (refs. 23 and 24). The geometric optic approach is attractive due to its simplicity. It also affords a straightforward explanation of the physical phenomenon.

The scope of this paper does not permit in-depth discussion of analytical techniques developed for analysis of MDRs. In simple terms, the MDRs can be explained as follows. When a sphere is placed in an electromagnetic field, the distribution of intensities of the field components inside it follows solutions of the scalar Helmholtz equation in spherical coordinates (refs. 12 and 13). These intensity distributions are also called eigenfunctions or modes. Due to the fact that geometry and material properties of the sphere are included in the equations resulting from solutions of the Helmholtz equation it is natural that these parameters, because they depend on the sphere alone, would affect the distribution of the intensities inside the sphere. Moreover, the sphere acts as a resonator by responding more strongly to some electromagnetic fields than to others. This phenomenon with applications to dielectric resonators and spheres in particular is described by Collin (ref. 25). Reference 25 also explains how part of the energy from the incident electromagnetic field is being stored in resonant modes inside the sphere. A conclusion that could be drawn is that the strength of resonances depends not only on geometry and material properties of spheres but on the spectrum of the incident electromagnetic field.

In the optical range of the electromagnetic spectrum, the trapping of energy inside optical microspheres can be explained in terms of total internal reflections (refs. 21 and 26). The coupled portion of the light that enters the sphere stays inside it, provided that the refractive index of the sphere is larger than that of its surrounding medium. The total internal reflection coupled with the matching conditions results in a resonance of certain wavelengths of the incident light inside the sphere. The trapped wavelengths and their strength are theoretically determined by the solutions of Maxwell's equations in the spherical coordinates inside the sphere and are expressed in terms of their radial and angular components or modes (ref. 12).

Thus, solutions of the scalar Helmholtz equation in spherical coordinates come in a form of so called eigenfunctions for the radial, azimuthal, and polar coordinates. These radial, azimuthal, and polar eigenfunctions are associated with the radial $(n)$, polar $(l)$, and azimuthal $(m)$ mode numbers (ref. 17). The radial modal number $n$ gives the number of nodes of the intensity distribution in radial direction. The polar mode number $l$ is approximately the number of wavelengths packed along the circumference of the resonator traveling along the equatorial belt of the sphere. For any value of $l, m$ varies as $|m| \leq l$. In a perfect sphere with uniform index of refraction, all $m$ modes have the same resonant wavelength as the $l$ modes as $m=l$. Furthermore, sets $l$ and $m$ are associated with angular positions $\theta$ and $\varphi$. The angles are measured in two orthogonal planes passing through the center of the sphere. For a given radius $r$, spherical components with the same values of angles $\theta$ and $\varphi$ have identical values. This is called degeneracy in the solution of Maxwell's equations in spherical coordinates (refs. 12 and 13).

In practical applications of MDRs the optical resonances are excited by coupling light into the sphere from a tunable laser at grazing angles. Several methods can be used to launch the light into the sphere in such a fashion. For example, a common approach in sensor applications is the side-coupling of the laser light using a single mode optical fiber (refs. 6, 7, and 24). As shown in figure 1 the optical fiber serves as an input-output conduit. As the laser is frequency tuned across a range, the optical resonances of the sphere are observed as sharp dips in the transmission spectrum through the fiber.

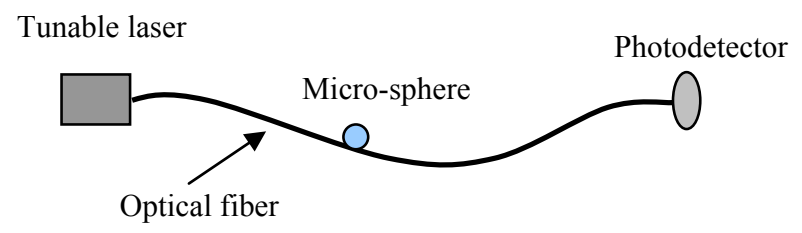

Figure 1.-Interrogation of optical resonances of a microsphere by side-coupled optical fiber. 


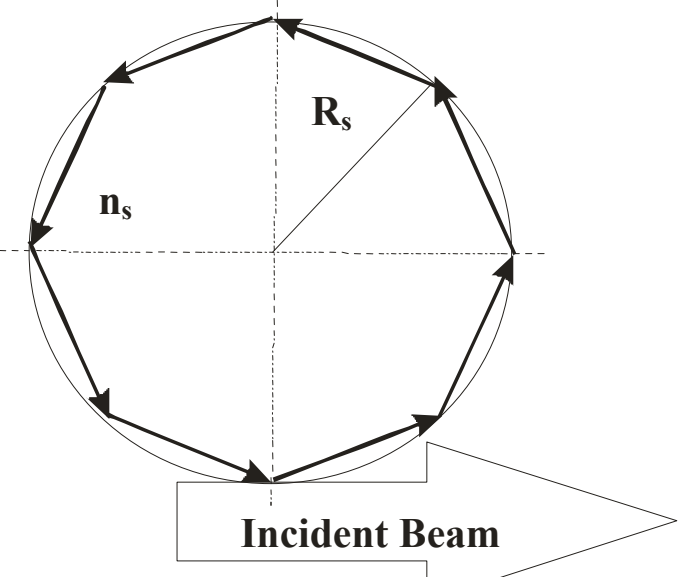

Figure 2.-Principle of Morphology Dependent Resonances.

Figure 2 demonstrates multiple reflections inside the microsphere of radius $R_{S}$ and refractive index $\mathrm{n}_{\mathrm{S}}$, once the light is coupled into it. For $\left.\left.R_{S}\right\rangle\right\rangle \lambda$, where $\lambda$ is the wavelength of light, the approximate resonance conditions within the sphere and the spacing, $\Delta \lambda_{l}$, between two consequent modes $l$, are respectively (ref. 24)

$$
2 \pi n_{s} R_{s}=l \lambda
$$

and

$$
\frac{\Delta \lambda_{l}}{\lambda} \approx \frac{\lambda}{2 \pi n_{S} R_{S}}
$$

Thus, a glass microsphere with a $200 \mu \mathrm{m}$ radius should generate, at the wavelength $\lambda=1300 \mathrm{~nm}$, resonances with a periodicity that satisfies $\Delta \lambda_{l} / \lambda \approx 10^{-3}$, and as a result will have a free spectral range of $\Delta \lambda_{\mathrm{FSR}} \approx 1.3 \mathrm{~nm}$. However, experimentally observed resonances are more densely packed (ref. 27) as seen in figure 3 where they are observed as sharp dips. In this case, the laser light is side coupled into the $\sim 200 \mu \mathrm{m}$ radius glass sphere via a single mode optical fiber (as in fig. 1) and the transmission spectrum through the fiber is observed. Teraoka et al. (ref. 8) also demonstrated that a presence of aqueous environments led to shifts of resonances in dielectric microspheres.

The occurrence of closely located resonances is due to several factors including the fact that the incident beam has a certain cross section and, as shown in figure 4, also excites resonances (or modes) in azimuth planes tilted with respect to the polar plane. The dashed lines show maximum inclinations of those planes. In a perfect sphere, as was discussed above, the modes are degenerate. The spheres used in experiments are, however, imperfect and their radii and homogeneity of the refractive indexes are not uniform. Therefore portions of the incident beam coupled under an angle see different propagating conditions than the one coupled along the main azimuth plane. The imperfections in the spheres lead to removal of degeneracy and formation of multiple intermediate resonances (refs. 21 and 26). The order of resonances in figure 4 could be identified, according to convention, by their quantum numbers $(n, l, m-1)$, $(n, l, m)$, and $(n, l, m+1)$. 


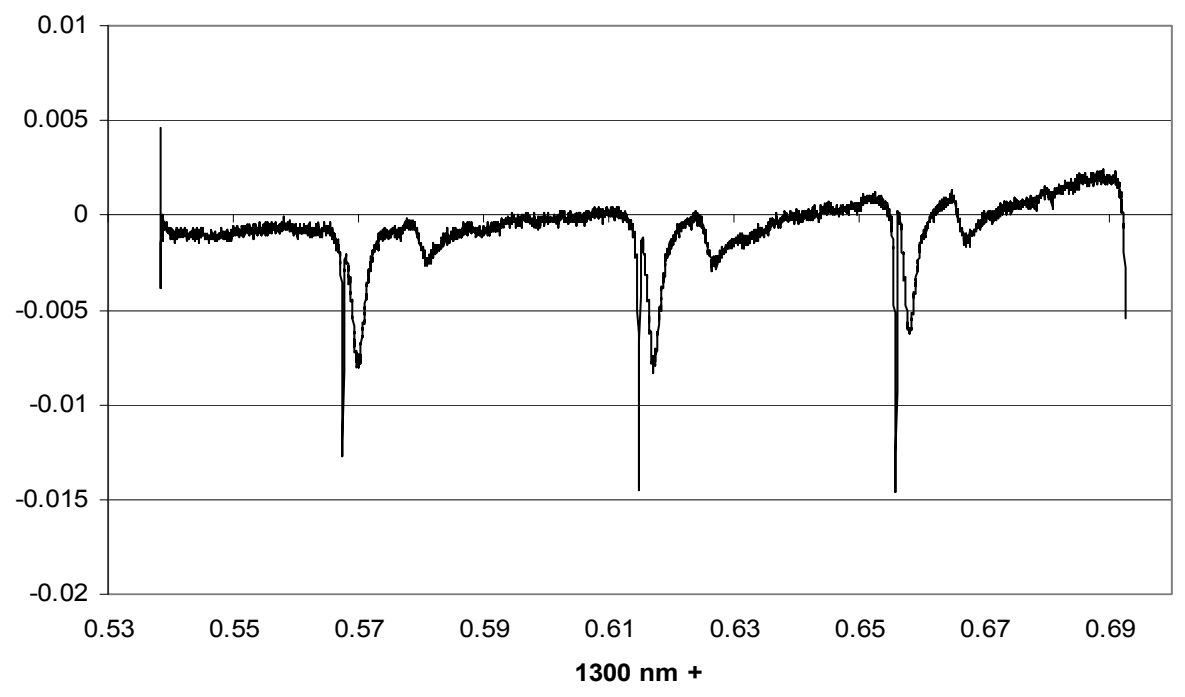

Figure 3.-Resonances in a microsphere with a radius of $\sim 200 \mu \mathrm{m}$.

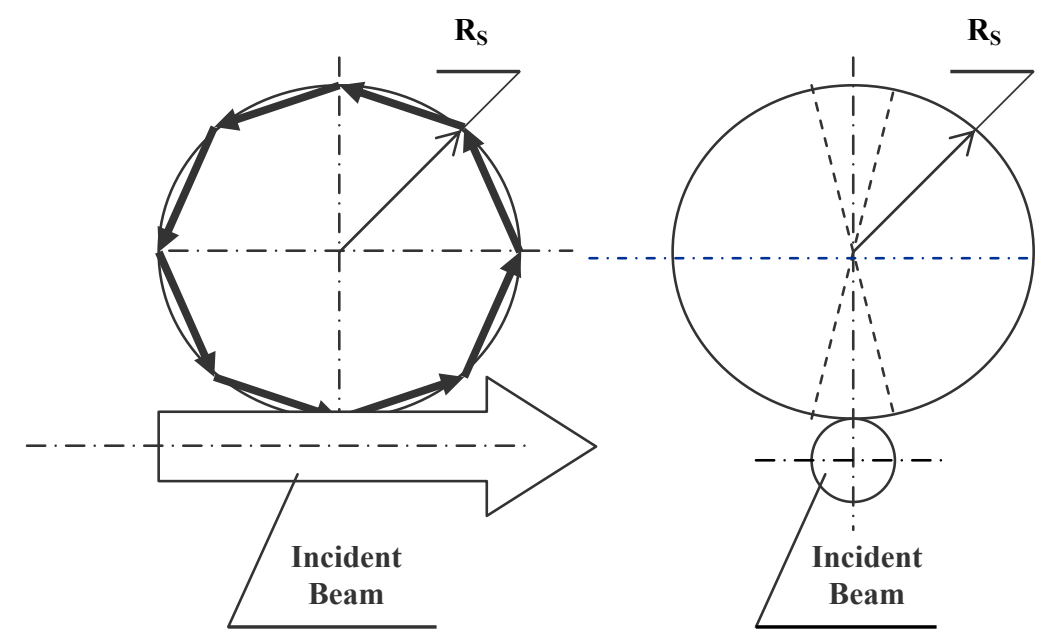

Figure 4.-Schematic explanation of formation of dense resonances in imperfect microspheres.

Previous studies of a Gaussian laser beam striking a sphere at a grazing incidence have demonstrated formation of MDRs in the sphere as well as an escape or return channel for the light leaving the sphere (refs. 28 and 29). Figure 5 shows schematically the formation of the return channel which could be used to extract resonances in the form of intensity peaks in the spectral domain rather than intensity dips as is shown in figure 3 (ref. 29).

Results of numerical calculations of light coupling into a microsphere are shown in figure 6 . The model used is derived from Maxwell's Equations with the Yee algorithm being chosen to simplify computational matching boundary conditions (ref. 30). Computations are done by the Finite-Difference Time-Domain (FD-TD) numerical method (refs. 31 and 32). Figure 6 shows distinct points of ingress and egress of the light into and from the sphere as well as the formation of a return channel. 


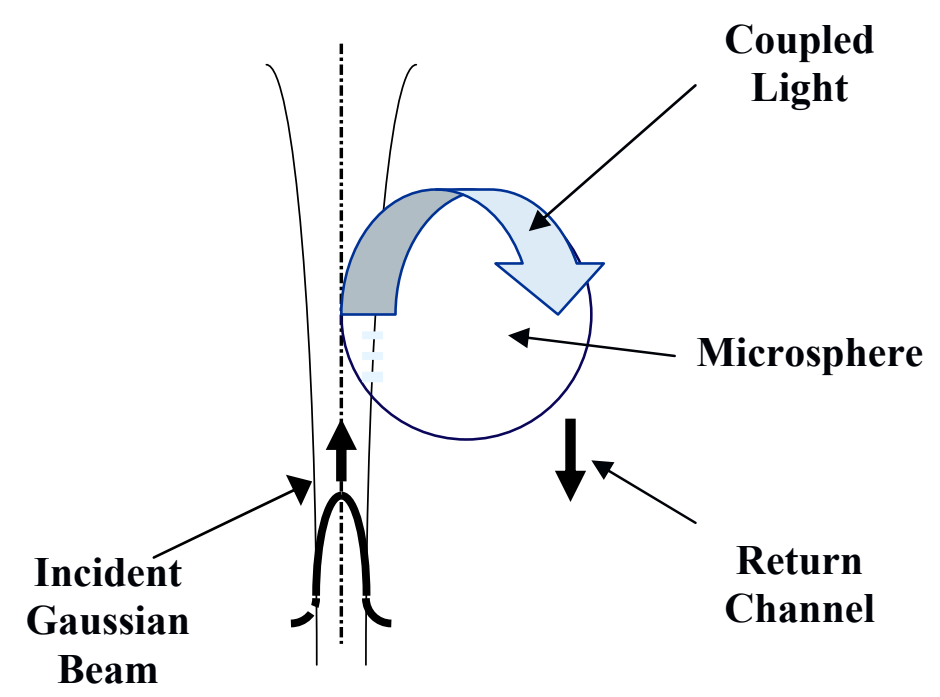

Figure 5.-Schematics of a Gaussian beam coupled into a microsphere.

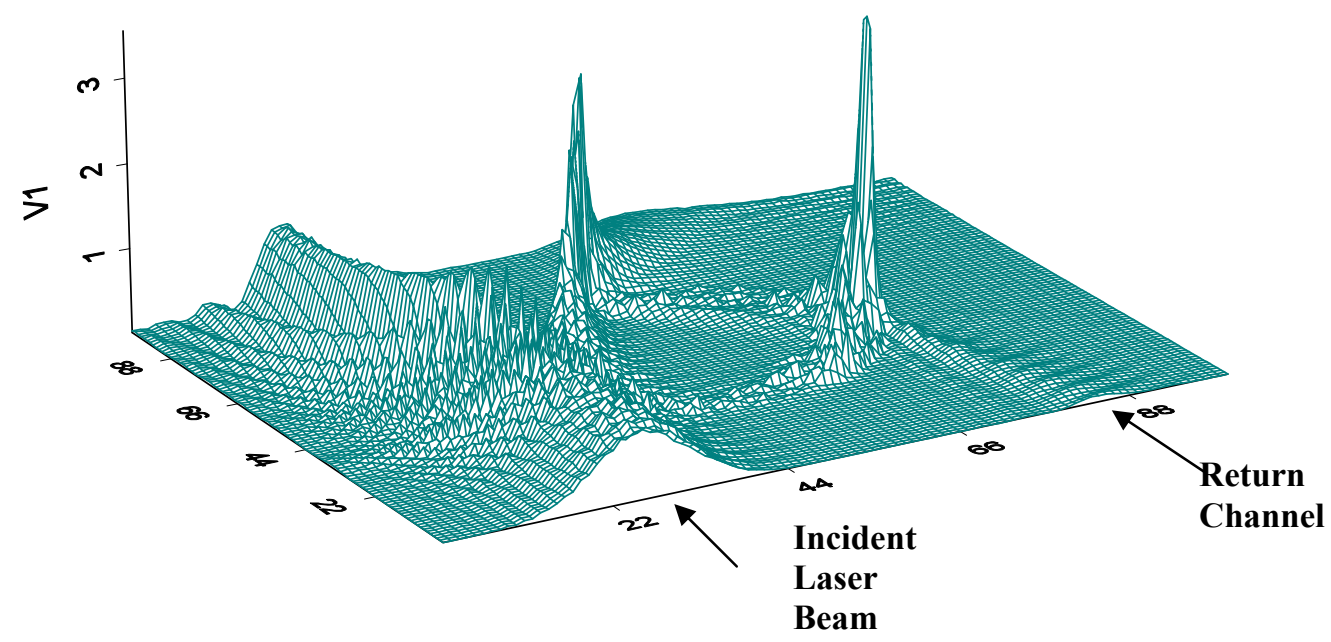

Figure 6.-Computational demonstration of morphology dependent resonances.

\section{Sensor Configurations}

\section{Excitation of Resonances in Microspheres}

MDRs in a sphere can be induced by an external electromagnetic field. At optical frequencies, an incident laser beam either is brought into a close contact with the sphere surface or strikes the sphere at a distance. In both cases resonances have been observed, reported, and used to study properties of microspheres and small droplets (refs. 33 and 35). As mentioned above, a commonly used approach for sensor applications is that shown schematically in figure 1 , where the incident beam is brought to the surface of a sphere via an optical fiber (refs. 6, 7, and 24). In order to facilitate the optical coupling between the sphere and the fiber, the section of fiber that is in contact with the sphere is tapered by either etching or heating and stretching it (ref. 36). Both cases are schematically presented in figure 7. 

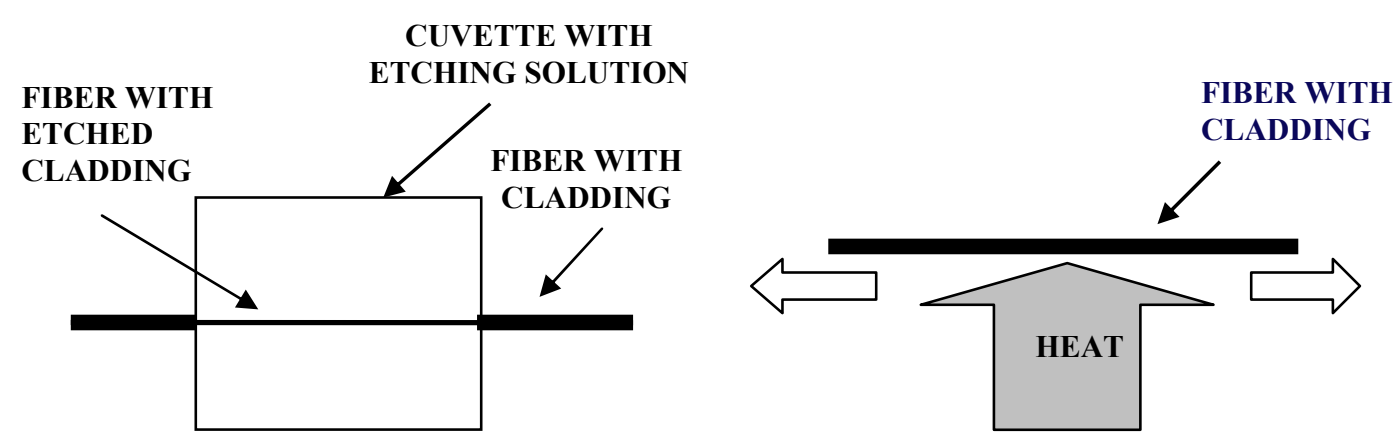

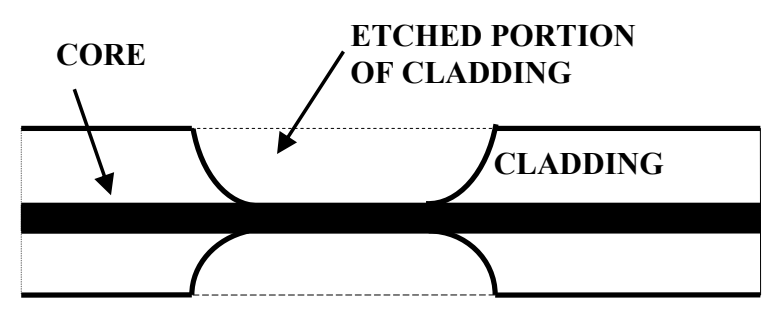

(a)
CORE

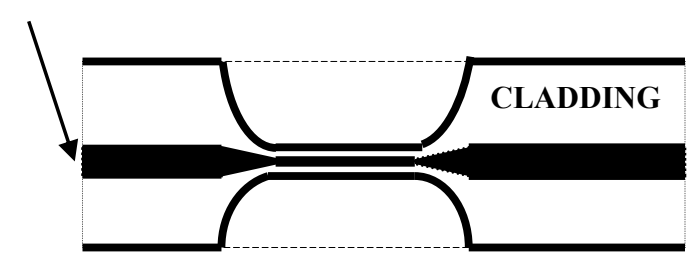

(b)

Figure 7.-Thinning of optical fiber by (a) etching and (b) heating and stretching.

In both cases, a portion of optical fiber is thinned out to extend its evanescent field beyond the fiber boundaries. The thinned section of the fiber has a diameter comparable with the fiber core. A small diameter of the thinned fiber permits positioning of a microsphere very close to the core of the fiber and provides a better coupling of light into the microsphere. However, the etched fiber tends to produce cleaner coupling because the core of a single mode fiber supports only one fundamental mode which has its evanescent field extending far beyond the core boundaries. The heated and stretched portion of a single mode fiber, on the other hand, despite its small diameter, has a cross section which consists of remnants of both core and cladding. The evanescent field, thus, is different from the etched fiber. The locations of resultant resonances in the spectral domain are also expected to be different. Figure 8 shows a picture of an etched fiber placed in a close proximity to a microsphere (ref. 37).

A typical conventional way of manufacturing microspheres involves melting the tip of a fiber until it forms a spheroid. The process results in a microsphere on the tip of a fiber stem and permits an easy manipulation and positioning of the microsphere.

Steady and repeatable resonances have been obtained by placing microspheres on so called half-block couplers (refs. 38 and 39). A half-block coupler is an optical structure in which a piece of bent fiber is embedded in a glass block in such a way that a portion of the side of the fiber is exposed. That portion of the exposed fiber is polished in order to bring the core closer to the surface. The structure provides a stable positioning of the microsphere on the top of the polished section of the fiber. Figure 9 demonstrates a microsphere-half-block configuration. 


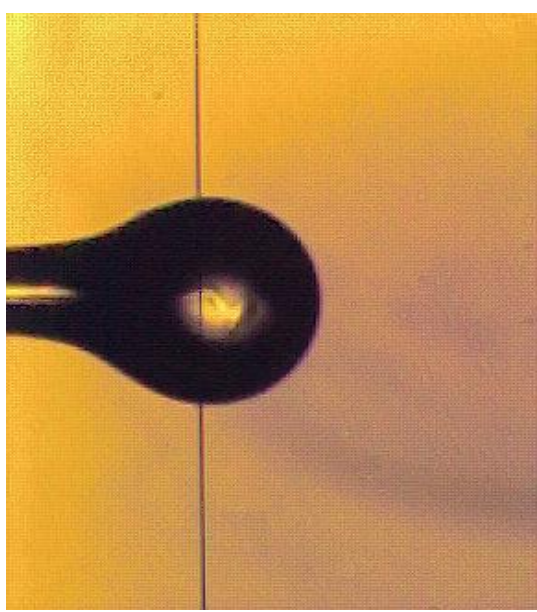

Figure 8.-Microsphere in a close proximity to etched fiber (adopted from reference 37).

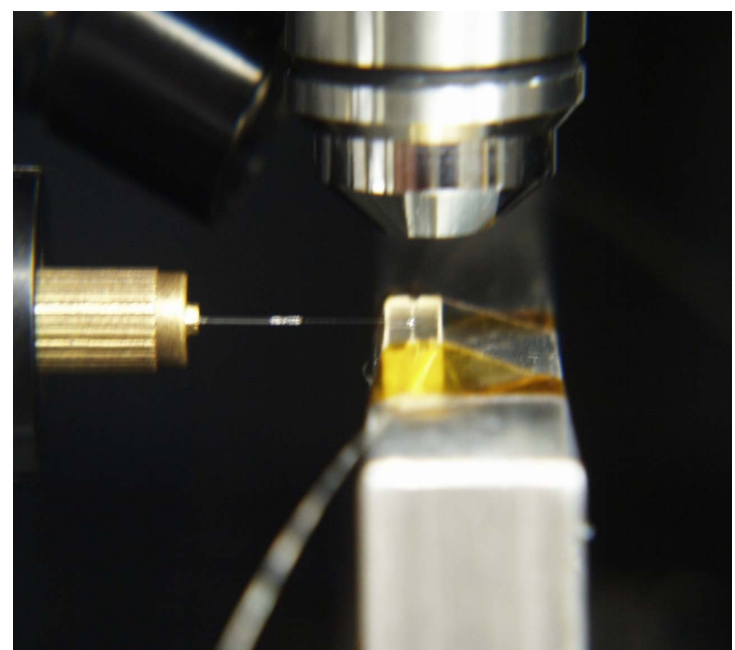

(a)

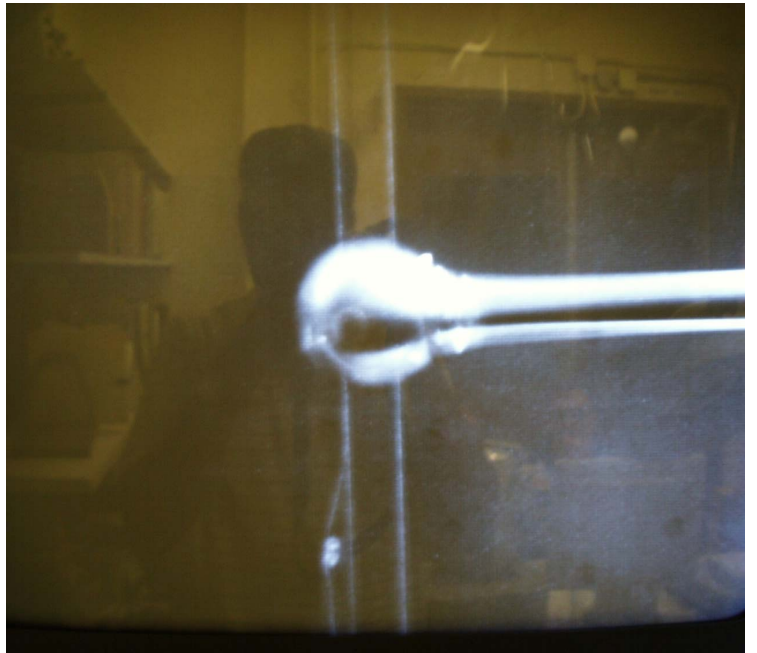

(b)

Figure 9.-Microsphere on the top of a half-block coupler. (a) Setup showing a microsphere on a fiber stem positioned on the coupler; (b) Microscope image of a microsphere on the top of $125 \mu \mathrm{m}$ single mode fiber.

\section{MDRs in Microspheres Over Extended Range of Wavelengths}

The conventional way of manufacturing microspheres by melting the tip of a fiber has a serious drawback. The technique cannot produce identical microspheres in a repeatable fashion. As a result, the MDR spectra from spheres manufactured by a seemingly identical method will not just have multiple intermediate resonances, but those resonances will vary from one microsphere to another. As the quality of microspheres improves an issue of the observable range of wavelengths comes into play.

The range of wavelengths over which the resonances in microspheres are observed is determined by the tunability of laser diodes used for these purposes. Typically, that range does not exceed $0.5 \mathrm{~nm}$ and is not sufficient to observe resonances associated with multiples of consecutive $l$ modes. To extend the laser diodes' operability range they are driven by a continuously changing current but at different temperatures. The operating range of a tunable laser diode may be extended by changing the operating temperature of the diode junction (ref. 27). Thus, tuning the laser continuously over fixed temperature intervals produces a broader range of operation. Such an approach, however, requires an accurate calibration. Figure 10 shows results of a calibration procedure that covers 5 temperature ranges and a wavelength range from about 1303.5 to $1306.25 \mathrm{~nm}$. The results are adopted from reference 27. 


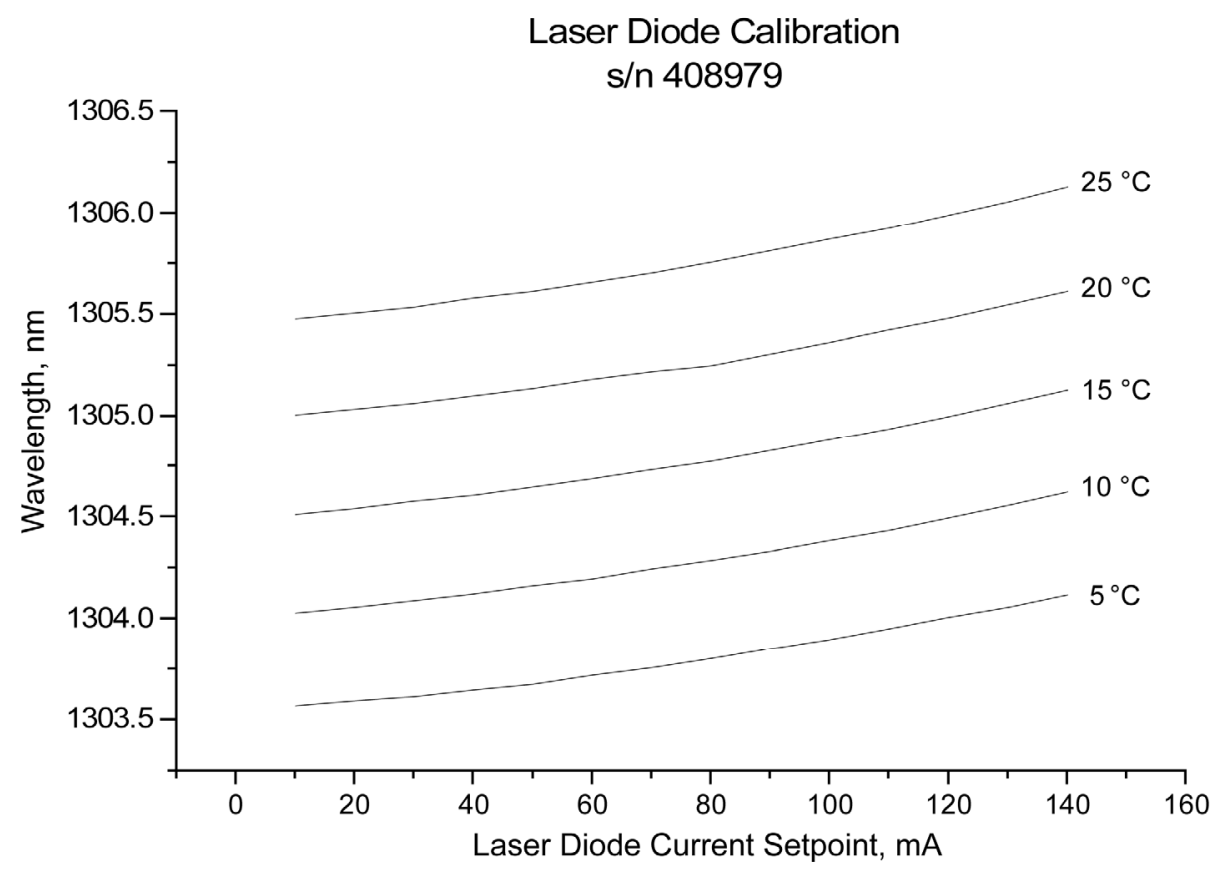

Figure 10._Laser diode calibration curves at different temperatures (adopted from reference 27).

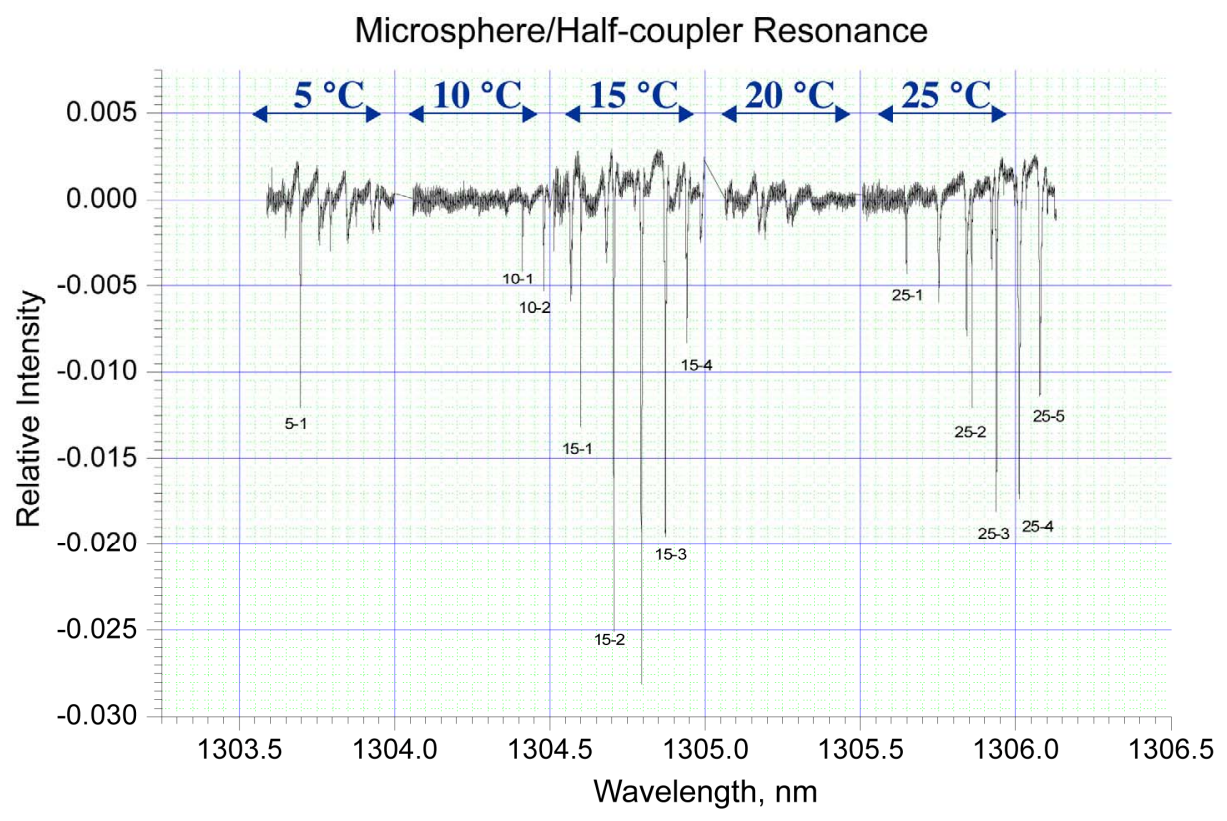

Figure 11.- Resonances over extended range of wavelengths (adopted from reference 27).

Resonances that typically occur over an extended range of wavelengths are shown in figure 11. It is obvious from figure 11 that the secondary resonances have a tendency to group around the fundamental ones. It is expected that as the uniformity of the microspheres improves the secondary resonances will gradually degenerate and coincide with the spectral positions of the fundamental resonances. 


\section{Sensor System}

Although experimental apparatus used to demonstrate various sensor applications may vary in their detail, general features of the typical setup are quite similar and may be represented by figure 12 which is adopted from reference 24 .

In this setup, a single mode optical fiber is used to carry light into and out of the sensor sphere. The setup consists of three main sub-systems (a) a tunable laser source with means to set, control, and monitor conditions of its junction temperature and current; (b) an optical structure with means to deliver the laser light to the sphere resonator and extract the resultant optical signal for further processing; and (c) a photodetector and appropriate electronics to detect and analyze resonances.

A computer and software are typically added to provide control of the instrumentation and data processing capability (including resonance dip detection and tracking). The output of the tunable laser, typically a distributed feedback (DFB) laser diode (refs. 24, 37, and 40 to 42) is coupled into a single mode optical fiber. The laser beam is split in two by an in-fiber beam splitter in such a manner that a small portion of the laser output is directed to a photodiode as a reference signal to monitor the laser intensity. The other fiber is in contact with the micro-sphere and serves as the optical input/output device. The output of this fiber is connected to a fast photodiode to monitor the transmission spectrum. The transmission spectrum through this fiber is normalized using the reference signal in order to remove the effects of laser output variations. The section of the fiber where it is in contact with the micro-sphere is tapered by either etching it or by heating and stretching as described above. For optical coupling between the fiber and the sphere, the evanescent fields of the two elements must overlap. If the optical driver is a DFB laser, it is typically tuned by ramping the current into it using a laser controller although temperature tuning or a combination of both is also possible. The laser controller can be driven by a function generator that provides an appropriate electronic signal profile (ramp function) into the controller. The output of the photodetectors is monitored by a Digital Acquisition card driven by a host computer. Several different methods can be used to manufacture the micro-spheres but a common approach, as is mentioned early, is to melt the tip of a small section of a suitable thickness of fiber made of the desired dielectric material to form a spheroid. The fiber forming and heating process may vary from one material to another (such as silica and polymeric materials). Details of micro-sphere manufacture for silica and polyhethylmethacralyte (PMMA) can be found in reference 41.

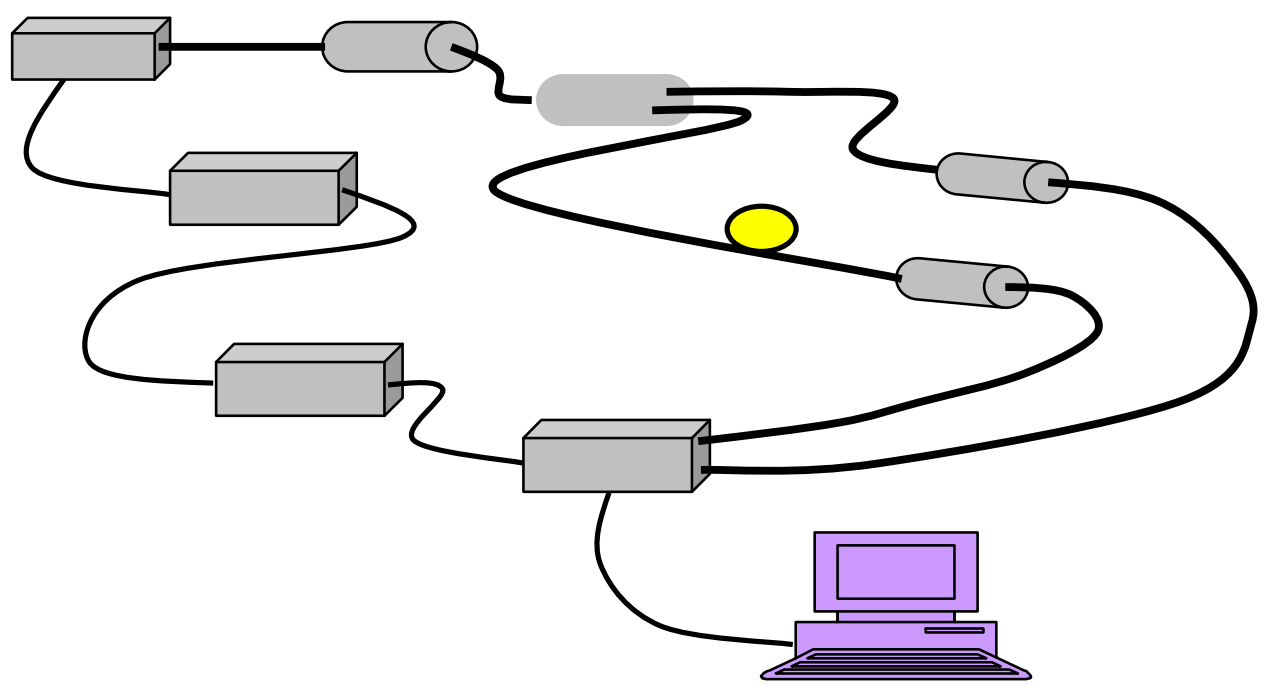

Figure 12.-Schematic of experimental setup (adopted from reference 24). 


\section{Aerospace Sensor Applications}

As discussed earlier, the high sensitivity of MDRs to the environmental conditions (due to the typically very high $Q$ factors) and their small size make them good candidates for a wide range of sensors including those for aerospace applications. Sensor systems used in aerospace applications belong mostly to either the vehicle control or health and performance monitoring category. In the first category are sensors that measure temperature, pressure, wall shear stress, position and other related parameters. The latter category includes force, strain, and acceleration sensors, among others.

\section{Temperature}

The temperature tuning of MDRs of a sphere was first demonstrated with a view of fast optical switching (ref. 43). The conjugate polymer coated sphere was optically pumped by a diode laser and the resulting resonance shifts were attributed to the thermal effect. In this study, the micro-sphere serves as an active element to perform the optical switching. Later, an MDR-based temperature sensor concept was demonstrated by Guan et al. (ref. 37) who used silica spheres to carry out measurements in air and water. As indicated by equation (1), a perturbation in both the radius and the refractive index of the sphere sensor will lead to an MDR shift. Hence,

$$
\frac{\Delta \lambda}{\lambda}=\frac{\Delta n_{s}}{n_{s}}+\frac{\Delta R_{s}}{R_{s}}
$$

In the MDR-based temperature sensor, changes in temperature lead to changes in both the radius $R_{S}$ due to the thermal expansion and refractive index $n_{s}$ due to the thermo-optic effect. Therefore, both terms on the right side of equation (3) contribute to the MDR shift, $\Delta \lambda$, in response to a temperature change of $\Delta T$;

$$
\frac{\Delta \lambda}{\lambda} \approx \alpha \Delta T+\beta \Delta T
$$

Here, $\alpha$ is the thermal expansion coefficient and $\beta$ is the thermo-optic coefficient of the microsphere. For spheres made of silica and conventional glass, the thermo-optic effect is larger than that of the thermal expansion. However, both effects are significant and need to be taken into account (ref. 37).

In the temperature sensor study described in reference 37, the experiments were carried out both in air and water. The silica microspheres (in the diameter range 150 to $400 \mu \mathrm{m}$ ) on a stem were placed inside a test cell along with an etched (in dilute hydrofluoric acid) section of the input/output optical fiber. The spheres were made by melting the tip of a silica optical fiber (cladding included) using a micro-flame torch. The diameter of the etched fiber was about 4 to $5 \mu \mathrm{m}$ permitting an easy coupling of light from the fiber into the microsphere. The temperature of the fluid in the cell was controlled by a heating plate that was placed at the roof of the cell. For comparison, the fluid temperature in the cavity was measured independently with thermocouples. As the temperature changed, the dips in the transmission spectrum corresponding to the resonances of the microsphere shifted allowing for the measurement of temperature (through the relationship given in equation (4).

Figure 13 shows sample results for water (a) and air (b). In both cases, the cell is first heated and then allowed to cool down and the corresponding time evolution of temperature is presented in the figure. The agreement between the MDR and thermocouple results is a good demonstration of the potential for an MDR-based micro-optical temperature sensor. In figure 13(b), the possibility of a distributed sensor concept is investigated where two micro-spheres are placed on the same optical fiber, close to one another. The software first recognizes two distinct resonance dips, one from each sphere, and then tracks their shifts independently over time. 


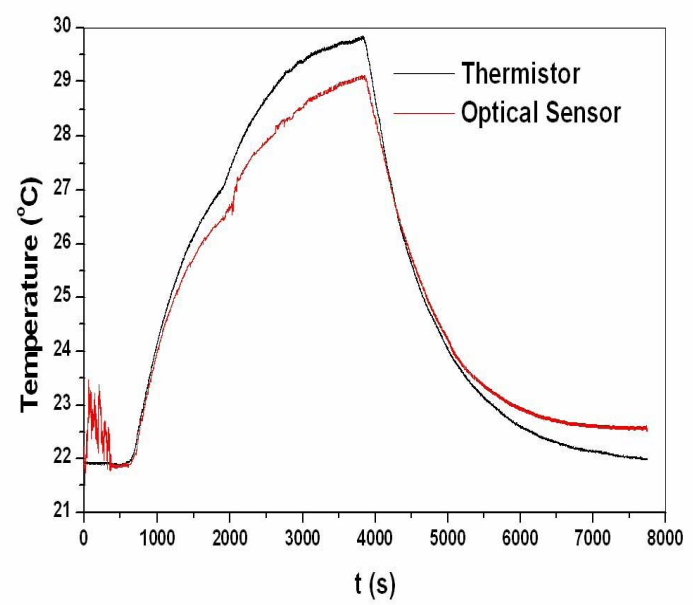

(a)

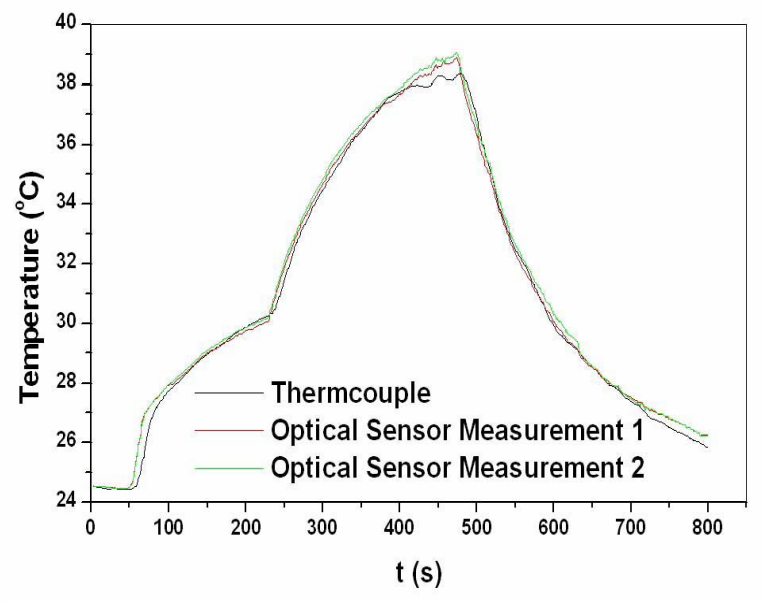

(b)

Figure 13.-Temperature measurements in (a) water and (b) air (adopted from reference 37).

\section{Force and Acceleration}

In an earlier investigation, the relationship between mechanical strain and MDRs of cylindrical micro-resonators was studied (ref. 44). In this work, a 1-mm length section of a 125- $\mu$ m diameter silica optical fiber was used as the optical resonator and it was strained using a PZT-driven vise that was attached to it. The beam of a tunable laser was focused on the side of the fiber and the MDR shifts were monitored through the elastically scattered spectrum. A resonance shift of $\sim 0.8 \mathrm{~nm}$ was observed for a strain of $\sim 5.8 \times 10^{-3}$. A similar study was carried out later using micro-spheres $(\sim 150 \mu \mathrm{m}$ diameter $)$ as the optical resonator (ref. 45). This time, a compressive force was applied to the resonator. The resonances of the silica sphere were excited by an external-cavity diode laser whose output was evanescently coupled to the sphere using a prism coupler. Strain-induced tuning ranges, $\Delta \lambda$, in excess of $0.3 \mathrm{~nm}$ were reported.

Along with the fact that very high $Q$-factors can be achieved with side-coupled spheres, these earlier studies raised the possibility of a high-sensitivity MDR- based micro-sphere force sensor. The MDRbased force sensors would also have the additional advantage of being compact with a small footprint and would lend themselves relatively easily to the development of distributed sensors. Further, since very small deformations of the microspheres (on the order of a $\mathrm{nm}$ ) can be detected, the force sensors would have essentially no moving parts. Recently, feasibility of such a sensor concept was demonstrated using silica (ref. 40) and Polymethyl-Methacrylate (PMMA) (ref. 41) micro-spheres. In the latter study, hollow PMMA spheres were used in addition to solid spheres in order to increase force sensitivity.

When a compressive force is applied along the polar direction of a sphere, as shown in figure 14, both its equatorial radius, $R_{s}$, and the refractive index $n_{s}$ (due to the induced stress field) are perturbed leading to a shift in the resonances as described in equation (3). The extent of each effect on $\Delta \lambda$ depends on the Young's modulus and elasto-optic properties of the sphere material. For both silica and PMMA, normal tensile stress produces $\Delta n_{s}<0$, thus stress tends to counteract the strain effect on $\Delta \lambda$.

The optical/electronic arrangement for the experiments described in references 40 and 41 was similar to that shown schematically in figure 12 . Force was exerted on the micro-sphere by using two hardened steel pads that compress the sphere in the direction perpendicular to the tapered optical fiber (see fig.14). The applied force was measured independently by a load cell that was placed in-line with one of the compression pads. The silica spheres were prepared using the same approach reported in reference 37. A different method was used for the PMMA spheres which involved purification of the polymer before forming the spheres. This process helped to reduce optical absorption which causes poor $Q$ values 
(ref. 41). The hollow PMMA spheres were obtained by injecting a controlled amount of air into the liquid polymer before depositing it on to the fiber stem. The $Q$ values for both solid and hollow PMMA spheres were close to $\sim 10^{6}$.

The study showed that the MDR-based force sensor can provide a linear response; for both silica and PMMS sphere resonators, the force- $\Delta \lambda$ relationship was linear. Figure 15 demonstrates the linear relationship between force and MDR shift for a solid silica sphere. It also shows that there is essentially no detectable hysteresis. Due to the smaller Young's modulus of PMMA, the polymeric sphere-based sensors can provide significantly improved sensitivity albeit with a reduced total force range. The results (see references 40 and 41) indicate that force resolutions of the order of $10^{-3} \mathrm{~N}$ are possible with solid silica spheres of diameter $\sim 300 \mu \mathrm{m}$. Using hollow PMMA spheres, the resolution can improve to $<10^{-5} \mathrm{~N}$. Once the universal calibration curve is obtained for a given sphere material and geometry, the only variable that determines the "gain" of such a sensor would be the sphere size.

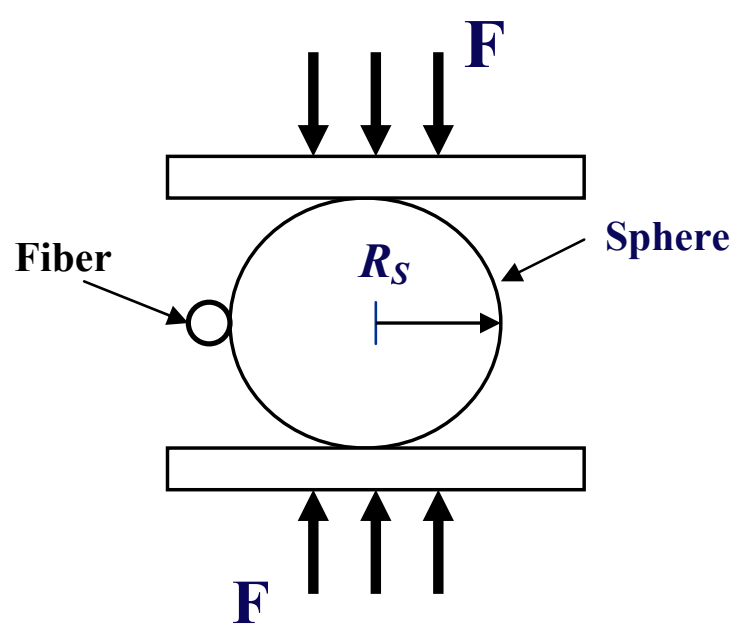

Figure 14.-Microsphere under compressive force.

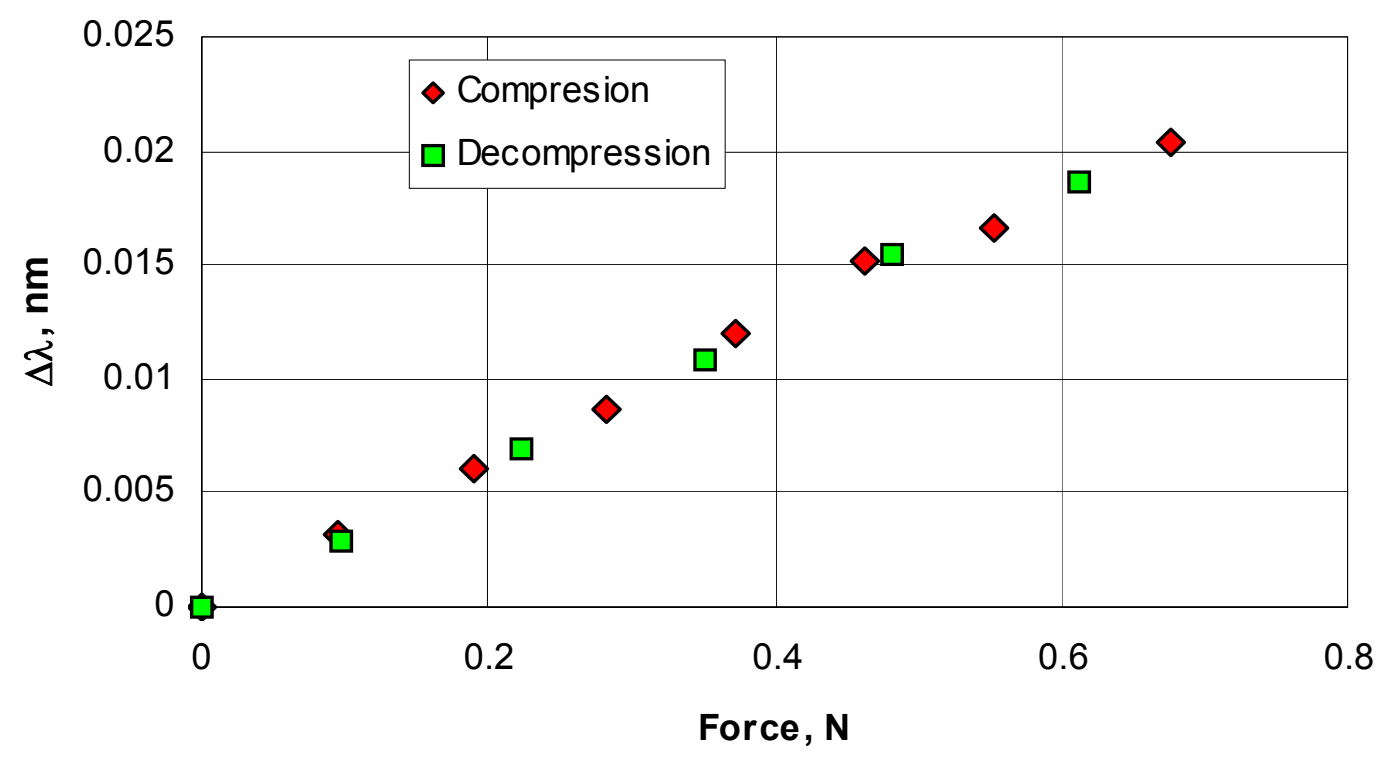

Figure 15.-MDR shift with applied force on a $430 \mu \mathrm{m}$-diameter solid silica (adopted from reference 40 ). 
In a recent study, Laine et al. (ref. 46) explored the application of MDR of spheres as an accelerometer. In this approach, the microsphere was fixed, through the fiber stem attached to it, in close proximity to a waveguide (as opposed to an optical fiber) which acted as the input/output device. The acceleration resulted in a change in the coupling distance between the waveguide and the sphere resulting in a perturbation of the resonance dip structure including the $Q$-factor. By monitoring the structure of a chosen resonance, acceleration measurements were achieved. A sensitivity of $<1 \mathrm{mg}$ at $250 \mathrm{~Hz}$ bandwidth was reported with a noise floor of $100 \mu \mathrm{g}$. The range and sensitivity of such an accelerometer can be controlled by changing the stem length, cross section and material.

\section{Pressure}

Ioppolo and Ötügen (ref. 42) carried out an analytical and experimental study of static pressure effects on the MDRs in order to determine the feasibility of a MDR-based pressure sensor. Combining elasticity equations for a sphere with Neumann-Maxwell elasto-optic expressions they developed analytical relations for resonance shifts of solid and hollow spheres due to changes in the static pressure of the surrounding medium. The analytical results were confirmed by experiments carried out with hollow PMMA spheres under varying atmospheric pressure. The study revealed that, as in the case of uniaxial force compression, the strain effect (sphere diameter change) dominates over that of stress (refractive index change). The study showed that for solid silica spheres large pressure variations are needed to realize a detectible resonance shift. Thus, for most aerospace applications, a silica-based pressure sensor would not be a good candidate. By the same token, atmospheric pressure changes are unlikely to interfere with resonances in silica microspheres used to sense other parameters such as force and temperature. However, hollow PMMA-based pressure sensors may be feasible due to the considerable $\Delta \lambda$ shifts with relatively moderate pressure change. As an example, figure 16 shows the experimentally and analytically obtained resonance shifts with pressure for a hollow $500 \mu \mathrm{m}$ radius PMMA. The inner-to-outer radius ratio for this case is $\mathrm{b} / \mathrm{a}=0.95$.

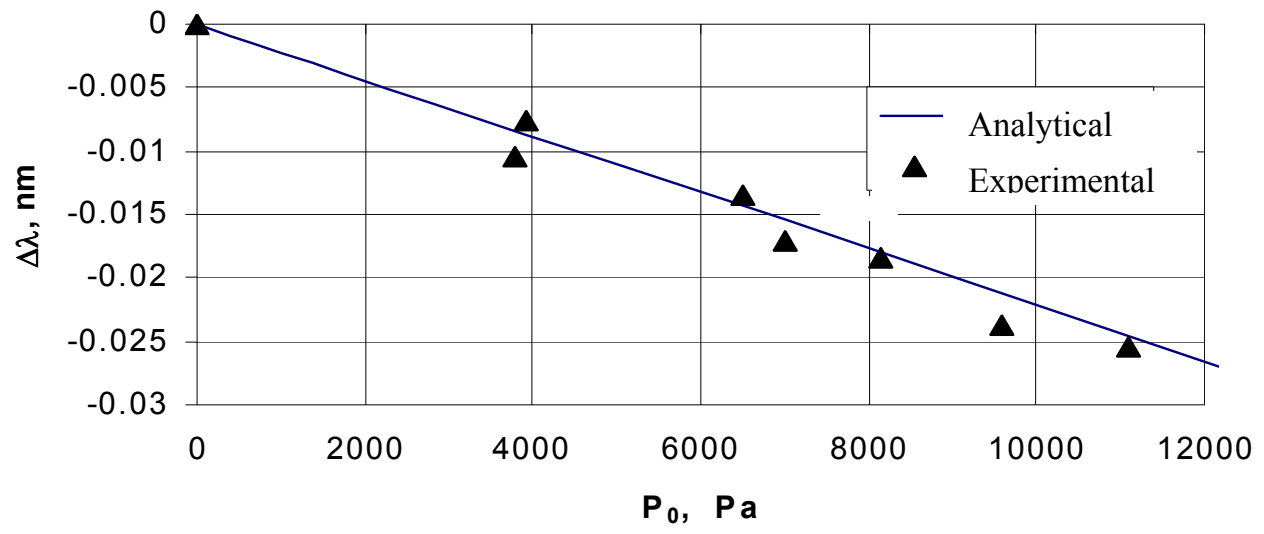

Figure 16.-Pressure tuning of WGM in hollow PMMA sphere with $R_{S}=500 \mu \mathrm{m}$ and $\mathrm{b} / \mathrm{a}=0.95$ (adopted from reference 42 ). 


\section{Concentration}

Das et al. (ref. 24) carried out a theoretical analysis to investigate the effect of species concentration of the surrounding fluid on the resonances of microspheres. They used a heuristic approach combining geometric optics with simple electromagnetic equations for wave reflection to obtain expressions that describe sphere resonances more accurately taking into account secondary effects not included in equation (1). Equation (1) represents the first order approximation of the resonances of a sphere taking into account only some of the sphere morphology. In fact, there are additional secondary effects that influence the resonance positions including the refractive index of the fluid wetting the sphere surface. A simple, heuristic explanation of this effect is as follows. Each time the light is reflected at the inner surface of the sphere due to total internal reflection, the reflected wave experiences a phase delay which is a function of the light wavelength and incidence angle as well as the fluid-to-sphere refractive index ratio. Therefore, even if the morphology of the sphere remains constant, changes in the fluid refractive index will result in a change of the total round trip traveled by the light ray trapped in the sphere thereby inducing a resonance shift. The question addressed by reference 42 was, if other environmental conditions that caused the first order effect (such as temperature) remained constant, could changes in the refractive index of the fluid (caused, for example, by changes in the chemical composition of the fluid) be detected by monitoring shifts of the resonances? Conversely, if these changes in the composition of the fluid were significant, would they interfere with the measurement of another parameter of the surrounding? The study showed that the resonances were not sensitive enough to refraction index change in gas media. However, they may be sufficiently sensitive in liquids for the possible development of optical sensors. These analytical results were confirmed through experiments where concentration of various salts was successfully measured in water (ref. 42).

\section{Discussion and Conclusions}

Several studies have been undertaken in the past few years exploring the application of MDRs of dielectric spheres for micro-optical sensors in aerospace applications. Proof-of-concept studies have shown that sensors for temperature, force, acceleration and pressure are feasible, and several more applications are currently under investigation (wall shear stress, strain, etc). The unusually high $Q$-factors that can be achieved by side coupling of light into the dielectric spheres allows for measurement sensitivities that may far exceed those of more conventional sensors, both mechanical and optical, including ones based on a Bragg grating. The small size of the sensors (with typical sphere sizes in the range from 100 to $1000 \mu \mathrm{m}$ ) is also attractive in terms of spatial resolution of measurements and in terms of being able to pack a large number of different types of sensors inside a small volume. Further, the fiber coupling approach may allow for the development of distributed sensors with multiples of spheres on a single optical fiber with one laser at its input and one optical detector at its output. Another potential application of MDRs is in smart structures where micro-spheres attached to optical fiber can be embedded inside structures acting both as reinforcements (fiber) and as sensors to monitor the health of the structure. Recent studies show that sensors with dielectric materials other than silica are also possible without a significant compromise in the $Q$-factors. Such materials (typically polymers) may be used to optimize the mechanical (such as Young's modulus and Poisson ratio), opto-elastic and thermo-optic properties of the sphere for different applications and sensing ranges. A common polymeric material that is easy to manufacture (as a sphere) is PMMA as demonstrated in the recent studies. However, in order to reduce optical absorption (and, hence increase $Q$-factor), the commercially available PMMA has to be purified.

Although MDR-based micro-sensors have been demonstrated at the proof-of-concept level in the laboratory environment, practical challenges do exist and they must be overcome before such sensors become available for use in the field. Selectivity and sensor ruggedness are two of the more significant challenges. The morphology of the dielectric spheres is affected by a number of environmental parameters and if multiples of them are varying at the same time, it would be difficult to isolate the desired effect for measurement. From a selectivity point of view, the MDR-based thermometry would be 
perhaps one of the more robust sensors. In aerospace applications, the other typical environmental conditions that are likely to vary are the static pressure and the concentration of trace elements in the air. But the recent studies discussed in this paper reveal that these two effects on the WGMs tend to be small compared to that of temperature. On the other hand, the effect of temperature on other sensor applications can be significant, even prohibitive. Methodologies need to be developed in order to suppress (appropriate material choice, etc) or eliminate/offset the temperature effect (independent measurement of temperature, for example). The problem with sensor robustness can be addressed by appropriately encapsulating the sphere together with the section of the optical fiber that is in contact with it. Encapsulation would also prevent liquid and solid particulate adsorption on the surface of the sphere causing unwanted WGM shifts. For the encapsulation approach to be successful, several conditions have to be met. For example, the refractive index of the encapsulating material has to be significantly smaller than that of the sphere so that high $Q$-values can be achieved with small scattering losses through the interface. At the same time, the elastic modulus and thermal expansion coefficients of the encapsulating and sphere materials have to be very close in order to minimize spurious WGM shifts due to material mismatch (caused by mechanical or thermal stress). Also, the interface between the sphere and the encapsulating material has to be smooth and of high quality to prevent scatter at the interface (which would result in reduced $Q$-values).

A majority of the challenges described above are essentially design and manufacturing challenges and are likely to be successfully addressed over time. Therefore, MDRs hold great promise in the future development of aerospace sensors.

\section{References}

1. Gorodetsky, M.L., Savchenkov, A.A., and Ilchenko, V.S., "Ultimate Q of Optical Microsphere Resonators," Opt. Lett. vol. 21, no. 7, 1996, pp. 453-455.

2. Belici, T., Isci, S., Kurt, A., and Serpenqüzel, A., "Microsphere-Based Channel Dropping Filter With an Integrated Photodetector," IEEE Photon. Technol. Lett., vol. 19, no. 2, 2004, pp. 476-478.

3. Ilchenko, V.S., Savchenko, A.A., Matsko, A.B., and Maleki, L., "Whispering-Gallery-Mode Electro-Optic Modulator and Photonic Microwave Receiver," J. Opt. Soc. Am. B, vol. 20, no. 2, 2003, pp. 333-342.

4. Matsko, A.B., Savchenko, A.A., Stekalov, D., Ilchenko, V.S., and Maleki, L., "Review of Applications of Whispering-Gallery Mode Resonators in Photonics and Nonlinear Optics," IPN Progress Report 42-162, 2005, URL: http://tmo.jpl.nasa.gov/progress_report/42-162/162D.pdf [cited 10 October 2007].

5. Little, B.E., Chu, S.T., Absil, P.P., Hryniewicz, J.V., Johnson, F.G., Seiferth, F., Gill, D., Van, V., King, O., and Trakalo, M., "Very High-Order Microring Resonator Filters for WDM Applications," IEEE Photon. Technol. Lett., vol. 16, no. 10, 2004, pp. 2263-2265.

6. Vollmer, F., Broun, D., Libchaber, A., Khoshsima, M., Teraoka, I., and Arnold, S., "Protein Detection by Optical Shift of a Resonant Microcavity," Appl. Phys. Lett., vol. 80, no. 21, 2002, pp. 4057-4059.

7. Arnold, S., Khoshsima, M., Teraoka, I., Holler, S., and Vollmer, F., "Shift of Whispering-Gallery Modes in Microspheres by Protein Absorption," Opt. Lett., vol. 28, no. 4, 2003, pp. 272-274.

8. Teraoka, I., Arnold, S., and Vollmer, F., "Perturbation Approach to Resonance Shifts of Whispering-Gallery Modes in a Dielectric Microsphere as a Probe of Surrounding Medium," JOSA B, vol. 20, no. 9, 2003, pp. 1937-1946.

9. Lee, K.M., Leung, P.T., and King, K.M., "Dyadic Formulation of Morphology-Dependent Resonances. I. Completeness Relation,” J. Opt. Soc. Am. B, vol. 16, no. 9, 1999, pp. 1409-1417.

10. Fuller, K.A., "Scattering and Absorption Cross Sections of Compounded Spheres. II. Calculations for External Aggregation," J. Opt. Soc. Am. A, vol. 12, no. 5, 1995, pp. 881-892.

11. Gouesbet, G., Maheu, B., and Gréhan, G., "Light Scattering From a Sphere Arbitrarily Located in a Gaussian Beam, Using a Bromwich Formulation," J. Opt. Soc. Am. A, vol. 5, no. 9, 1988, pp. 1427-1443.

12. Balanis, C.A., Advanced Engineering Electromagnetics, John Wiley \& Sons, New York, 1989, Chapter 10.

13. Stratton, J.A., Electromagnetic Theory, McGraw-Hill, New York, 1941, Chapter VII.

14. Collin, R.E., The Field Theory of Guided Waves, 2nd ed., IEEE Press, New York, 1991, pp. 114-121.

15. Morse, P.M. and Feshbach, H., Methods Theoretical Physics, Part II, McGraw-Hill, 1953, pp. 1462-1469. 
16. Balanis, C.A., Advanced Engineering Electromagnetics, John Wiley \& Sons, New York, 1989, Section 3.4.3.

17. Kippenberg, T.J.A., "Nonlinear Optics in Ultra-High-Q Wispering Gallery Optical Microcavities," Doctoral Dissertation, California Institute of Technology, 2004, Chapter 2, URL: http://www.its.caltech.edu/ tjk/TJKippenbergThesis.pdf [cited 25 March 2008]

18. Guimarães, L.G., and Nussenzveig, H.M., "Theory of Mie Resonances and Ripple Fluctuations," Opt. Communication, vol. 89, no. 5-6, 1992, pp. 363-369.

19. Guimarães, L.G., "Theory of Mie Caustics," Opt. Commun., vol. 103, 1993, pp. 339-344.

20. Johnson, B.R., "Theory of Morphology-Dependent Resonances: Shape Resonances With Width Formulas," $J$. Opt. Soc. Am. A, vol. 10, no. 2, 1993, pp. 343-352.

21. Vollmer, F., "Resonant Detection of Nano to Microscopic Objects Using Whispering Gallery Modes," Doctoral Dissertation, The Rockefeller University, 2004, URL: http://www.rowland.harvard.edu/rjf/vollmer/images/vollmer_thesis.pdf [cited 20 March 2008]

22. Arnold, S., "Microspheres, Photonic Atoms and the Physics of Nothing," American Scientist, vol. 89, 2001, pp. 414-421.

23. Roll, G. and Schweiger, G., "Geometrical Optic Model of Mie Resonances," J. Opt. Soc. Am. A, vol. 17 , no. 7, 2000, pp. 1301-1311.

24. Das, N., Ioppolo, T., and Ötügen, V., "Investigation of Micro-Optical Species Concentration Sensor Concept Based on Whispering Gallery Mode Resonators," AIAA Paper no. 2007-1199, January 2007.

25. Collin, R.E., The Field Theory of Guided Waves, 2nd ed., IEEE Press, New York, 1991, Section 6.6.

26. Ching, E.S.C., Leung, P.T., and Young, K. "The Role of Quasinormal Modes," Optical Processes in Microcavities, eds., R.K. Chang and A.J. Campillo, World Scientific, Singapore, 1996, pp. 1-75.

27. Adamovsky, G., Juergens, G.R., Wanner, J., and Floyd, B., "Morphology Dependent Resonances in Microspheres Over Extended Range of Wavelengths," SPIE Great Lakes Photonics Symposium GLPS’06, Presentation GL102-12, Dayton OH, June 15, 2006.

28. Serpenqüzel, A., Arnold, S., Griffel, G., and Lock, J.A., "Optical Spectroscopy of Microcavities," Quantum Optics and the Spectroscopy of Solids, eds., T. Hakioglu and A.S. Shumovsky, Kluwer Academic Publishers, 1997, pp. 237-248.

29. Serpenqüzel, A., Arnold, S., Griffel, G., and Lock, J.A., "Enhanced Coupling to Microsphere Resonances With Optical Fibers," J. Opt. Soc. Am. B, vol. 14, no. 4, 1997, pp. 790-795.

30. Yee, K. S., "Numerical Solution of Initial Boundary Value Problems Involving Maxwell's Equations," IEEE Trans. Antennas and Propagation, vol. 14, 1966, pp. 302-307.

31. Adamovsky, G. and Ida, N., "Laser Beam Propagation Through Inhomogeneous Media With Shock-Like Profiles: Modeling and Computing," Optical Technology in Fluid, Thermal, and Combustion Flow III, S.S. Cha, J.D. Trolinger, and M. Kawahashi, eds., Proceedings SPIE, vol. 3172, 1997, pp. 530-539.

32. Taflove, A., Computational Electrodynamics: the Finite-Difference Time-Domain Method, Artech House, Boston, 1995.

33. Barton, J.P., "Effects of Surface Perturbations on the Quality and the Focused-Beam Excitation of Microsphere Resonance," J. Opt. Soc. Am. A, vol. 16, no. 8, 1999, pp. 1974-1980.

34. Lai, H.M., Leung, P.T., Poon, K.L., and Young, K., "Electrostrictive Distortion of a Micrometer-Sized Droplet by a Laser Pulse," J. Opt. Soc. Am. B, vol. 6, vol. 12, 1989, pp. 2430-2437.

35. Huston, A.L., H.-B. Lin, H.-B., Eversole, J.D., and Campillo, A.J., "Effect of Bubble Formation on Microdroplet Cavity Quality Factors," J. Opt. Soc. Am. B, vol. 13, no. 3, 1996, pp. 512-531.

36. Lefevre-Seguin, V., Knight, J.C., Sandoghar, V., Weiss, D.S., Hare, J., Raimond, J.-M, and Haroche, S., "Very High Q Whispering-Gallery Modes in Silica Microspheres for Cavity-QED Experiments," Optical Processes in Microcavities, eds., R.K. Chang and A.J. Campillo, World Scientific, Singapore, 1996, pp. 101-133.

37. Guan, G., Arnold, S., and Ötügen, V., "Temperature Measurements Using a Micro-optical Sensor Based on Whispering Gallery Modes," AIAA Journal, vol. 44, no.10, 2006, pp. 2385-2389.

38. Griffel, G., Arnold, S., Taskent, D., Serpenqüzel, A., Connolly, J., and Morris, N., "Morphology-Dependent Resonances of a Microsphere-Optical Fiber System,” Opt. Lett., vol. 21, no. 10, 1996, pp. 695-697.

39. Serpenqüzel, A., Arnold, S., and Griffel, G., "Excitation of Resonances of Microspheres on Optical Fiber," Opt. Lett., vol. 20, no. 7, 1995, pp. 654-656.

40. Kozhevnikov, M., Ioppolo, T., Stepaniuk, V., Sheverev, V., and Ötügen, V., "Optical Force Sensor Based on Whispering Gallery Mode Resonators," AIAA Paper No 2006-649, January 2006. 
41. Ioppolo, T., Kozhevnikov, M., Stepaniuk, V., Otugen, V., and Sheverev, V., "Performance of a Whispering Gallery Mode Resonator-Based Micro-Optical Force Sensor," AIAA Paper No 2007-1262, January 2007.

42. Ioppolo, T., and Otugen, M.V., "Pressure Tuning of Whispering Gallery Mode Resonators," J. Opt. Soc. Am. B, vol. 24 , no. 10,2007 , pp. $2721-2726$.

43. Tapalian, H.C., Laine, J.-P, and Lane, P.A., "Thermooptic Switches Using coated Microsphere Resonators," IEEE Photonics Technology Letters, vol. 14, 2002, pp. 1118-1120.

44. Huston, A.L. and Eversole, J.D., "Strain-Sensitive Elastic Scattering From Cylinders," Optics Letters, vol. 18, no 13, 1993, pp. 1104-1106.

45. Ilchenko, V.S., Volikov, P.S., Velichansky, V.L., Treussart., Lefèvre-Seguin, V., Raimond, J. -M, and Haroche, S., "Strain-Tunable High-Q Optical Microsphere Resonator," Optics Communications, vol. 145, nos. 1-6, 1998, pp. 86-90.

46. Laine, J.P., Tapalian, C., Little, B., and Haus, H., "Acceleration Sensor Based on High-Q Optical Microsphere Resonator and Pedestal Antiresonant Reflecting Waveguide Coupler," Sensors and Actuators A, vol. 93, no. 1, 2001, pp. 1-7. 


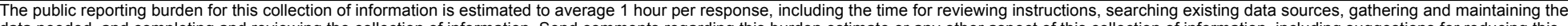

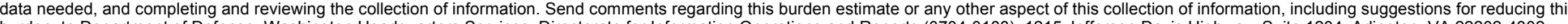

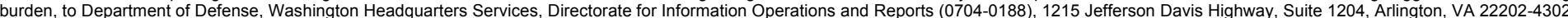

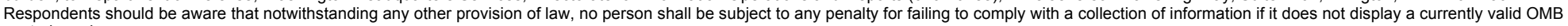
control number.

PLEASE DO NOT RETURN YOUR FORM TO THE ABOVE ADDRESS
1. REPORT DATE $(D D-M M-Y Y Y Y)$
2. REPORT TYPE
3. DATES COVERED (From - To)

01-03-2009

\section{TITLE AND SUBTITLE}

Technical Memorandum

Morphology-Dependent Resonances and Their Applications to Sensing in Aerospace

Environments

5b. GRANT NUMBER

NAG3-2679

5c. PROGRAM ELEMENT NUMBER

6. AUTHOR(S)

Adamovsky, G.; Otugen, M., V.

\section{5d. PROJECT NUMBER}

5e. TASK NUMBER

5f. WORK UNIT NUMBER

WBS 645846.02.07.03.03.03.01

\section{PERFORMING ORGANIZATION} REPORT NUMBER

E-16426

National Aeronautics and Space Administration

John H. Glenn Research Center at Lewis Field

Cleveland, Ohio 44135-3191

9. SPONSORING/MONITORING AGENCY NAME(S) AND ADDRESS(ES)

National Aeronautics and Space Administration

Washington, DC 20546-0001

10. SPONSORING/MONITORS
ACRONYM(S)
NASA
11. SPONSORING/MONITORING
REPORT NUMBER
NASA/TM-2009-215183

\section{DISTRIBUTION/AVAILABILITY STATEMENT}

Unclassified-Unlimited

Subject Categories: 35, 06, and 74

Available electronically at http://gltrs.grc.nasa.gov

This publication is available from the NASA Center for AeroSpace Information, 301-621-0390

\section{SUPPLEMENTARY NOTES}

Contains material published in the AIAA Journal of Aerospace Computing, Information, and Communication (JACIC), vol. 5, Oct. 2008.

\section{ABSTRACT}

This paper reviews recent developments in Morphology-Dependent Resonance (MDR)-based sensors for aerospace applications. The sensor concept is based on the detection of small shifts of optical resonances (also called the whispering gallery modes or WGM) of dielectric spheres caused by external effects. Recent developments in MRD-based micro-optical sensors for temperature, force, pressure, and concentration are discussed. In addition to the experimental configurations used in each type of prototype sensor, a brief overview is also given for analytical approaches to describe the sensor principle.

\section{SUBJECT TERMS}

Sensors; Measuring instruments; Optics; Optical resonance; Optical resonators

\begin{tabular}{|c|c|c|c|c|}
\hline 16. SECURI & ASSIFICATION & & 17. LIMITATION OF & 18. NUMBER \\
\hline $\begin{array}{l}\text { a. REPORT } \\
\text { U }\end{array}$ & $\begin{array}{l}\text { b. ABSTRACT } \\
\text { U }\end{array}$ & $\begin{array}{l}\text { c. THIS } \\
\text { PAGE } \\
\text { U }\end{array}$ & UU & $\begin{array}{c}\text { PAGES } \\
24\end{array}$ \\
\hline
\end{tabular}



